\title{
Sindrome serotoninérgico como reacción adversa infrecuente ante la sinergia entre Linezolid y Fentanilo: presentación de caso
}

\author{
Jose Leonel Zambrano-Urbano* \\ José Mauricio Ocampo-Chaparro** \\ Eduardo Lerma-Flórez ${ }^{* * *}$ \\ María Eugenia Casanova****
}

\begin{abstract}
*Residente. Medicina Interna. II año. Universidad Libre Seccional Cali. Colombia
**Médico familiar y Geriatra. Magister en epidemiología y gerontología social. Departamento de Medicina Interna. Grupo Interinstitucional de Medicina Interna (GIMI 1). Universidad Libre Cali, Colombia. Departamento de Medicina Familiar. Facultad de Salud, Universidad del Valle. Cali, Colombia.

***Médico anestesiólogo. Departamento de Anestesiología. Facultad de Salud. Universidad del Valle. Cali. Colombia.

****Médico Internista. Directora programa especialización en Medicina Interna. Departamento de Medicina Interna. Grupo Interinstitucional de Medicina Interna (GIMI 1). Universidad Libre Cali, Colombia.

Correspondencia: Doctor Jose Leonel Zambrano Urbano. Dirección: Cra. 35 \# 3a - 38 Barrio San Fernando Cali, Colombia. Teléfono: 3016130773. Correo electrónico: jose.zambrano@hotmail.com
\end{abstract}

\begin{abstract}
Resumen
El síndrome serotoninérgico es una condición potencialmente mortal causada por medicamentos que afectan el metabolismo de la serotonina o que actúan como agonistas directos del receptor de esta o una combinación de ambos. El síndrome da lugar a una variedad de manifestaciones mentales, autonómicas y neuromusculares, que pueden variar desde leves hasta potencialmente mortales. Se reporta el caso clínico de un paciente el cual desarrolló este síndrome por la coadministración y sinergismo de linezolid y fentanilo, con una gran variedad de características clínicas, desde las más sutiles, como cifras tensionales altas de difícil manejo mientras se encontraba bajo el efecto de sedoanalgesia, hasta las manifestaciones más floridas del síndrome posterior a la suspensión de esta. La asociación de estos medicamentos representa una etiología poco informada que puede favorecer la aparición del síndrome, mientras que el uso de benzodiazepinas puede enmascarar el cuadro dificultando su diagnóstico. MÉD.UIS.2020;33(3): 59-66
\end{abstract}

Palabras claves: Síndrome de la serotonina. Linezolid. Fentanilo.

\section{Serotonin syndrome as an infrequent adverse reaction to synergy between Linezolid and Fentanyl: case presentation}

\section{Abstract}

Serotonin syndrome is a life-threatening condition caused by medications that affect serotonin metabolism or that act as direct agonists for serotonin receptor or a combination of both. The syndrome gives rise to a variety of mental, autonomic, and neuromuscular manifestations, which can range from mild to life-threatening. We report a clinical case of a patient who developed this syndrome due to the co-administration and synergism of linezolid and fentanyl, with a wide variety of clinical characteristics, from the most subtle, such as high blood pressure levels difficult to manage while under the effect of sedoanalgesia, to the most florid manifestations of the syndrome after 48 hours of its suspension. The association of these drugs represents a poorly reported etiology that may favor the appearance of the syndrome, while the use of benzodiazepines may mask the condition, making its diagnosis difficult. MÉD.UIS.2020;33(3):59-66

Keywords: Serotonin syndrome. Linezolid. Fentanyl.

Artículo recibido el 12 de julio de 2020 y aceptado para publicación el 25 de octubre de 2020. 
¿Cómo citar este artículo?: Zambrano-Urbano J, Ocampo-Chaparro J, Lerma-Flórez E, Casanova $M$. Síndrome serotoninérgico como reacción adversa infrecuente ante sinergia entre Linezolid y Fentanilo: Presentación de caso. MÉD.UIS.2020;33(3):59-66. doi: 10.18273/revmed.v33n3-2020007

\section{Introducción}

El Síndrome Serotoninérgico (SS) es una condición potencialmente mortal, asociada con una mayor actividad serotoninérgica tanto en el sistema nervioso periférico (SNP) como en el sistema nervioso central (SNC), causada por medicamentos que afectan el metabolismo de esta hormona o que actúan como agonistas de los receptores delamisma'. Laserotonina es una hormona que actúa en el SNC modulando la atención, el comportamiento y la termorregulación y en el SNP, participa en la regulación de la motilidad gastrointestinal (principalmente por las células enterocromafines intestinales), la vasoconstricción y la broncoconstricción ${ }^{2}$. Dentro de su etiología está el empleo de medicamentos y en su mayoría, interacciones involuntarias entre diferentes fármacos $y$ en casos de envenenamiento intencional3,4. Los inhibidores de la monoaminooxidasa (selegilina, rasagilina, linezolid), inhibidores Selectivos de la Recaptación de Serotonina (ISRS) (fluoxetina, escitalopram, sertralina) y los inhibidores de la recaptación de serotonina y norepinefrina (duloxetina, venlafaxina) son los medicamentos más comúnmente asociados con el SS1,5. Así pues, puede ser el resultado de cualquier combinación de medicamentos que tenga el efecto neto de aumentar la neurotransmisión serotoninérgica (Ver tabla 1) ${ }^{6,7}$.

La incidencia del SS resulta difícil de establecer ${ }^{2,8}$. Este observa en todo el rango de grupos de edad, desde recién nacidos hasta adultos mayores, con una incidencia en aumento que probablemente represente el uso creciente de fármacos serotoninérgicos en la práctica clínica ${ }^{1,4}$. El porcentaje de adultos que reciben antidepresivos en los Estados Unidos casi se duplicó entre 1999 y 2010, pasando del 6\% al 10.4\%. Un estudio de cohorte retrospectivo de datos de las asociaciones "Veterans Health Administration" (VHA) e "Intercontinental Marketing Services PharMetrics Plus" en Estados Unidos que revisó los registros de la Administración de Salud de Veteranos de 2009-2013 mostró una incidencia de enfermedad del $0.23 \%$ en pacientes expuestos a medicamentos serotoninérgicos ${ }^{10}$. En 2016, el Sistema de Vigilancia de la Asociación Estadounidense de Centros de Control de Envenenamiento el cual recibe reportes de casos de los departamentos de emergencias, hospitalización y atención primaria, informó 54410 exposiciones a inhibidores selectivos de la recaptación de serotonina (ISRS; 43\% de los cuales fueron exposiciones únicas), con 102 muertes (siendo la novena causa más común de muerte por sobredosis de drogas en los Estados Unidos en este período), representando un aumento del $18 \%$ en los casos entre 2002 y 2016 y un aumento del $8 \%$ en el número de muertes ${ }^{11,12,13}$. Las grandes series de casos sugieren que se produce SS moderado en aproximadamente el $15 \%$ de las intoxicaciones con ISRS ${ }^{14}$. Aun así, se desconoce la verdadera incidencia de SS, habiendo varias razones para esto: que es una condición poco frecuente que no se detecta con facilidad en los ensayos clínicos controlados aleatorizados, además es poco reconocida e informada por el personal de salud (el $85 \%$ de los médicos generales no están familiarizados con la condición) y los casos leves a menudo se descartan o son autolimitados ${ }^{15,16}$. De todos los medicamentos, los ISRS son la clase más común implicada como etiología de este síndrome ${ }^{17}$. Usualmente (70\% de los casos), el SS resuelve con la deprescripción del medicamento dentro de las 24 horas posteriores a su ingesta; en el resto, el $40 \%$ requiere internación en terapia intensiva, y el $25 \%$ requiere intubación endotraqueal ${ }^{18}$. La mortalidad se ve entre el 2 al $12 \%$ de los casos ${ }^{2}$.

Su diagnóstico es netamente clínico ${ }^{4}$. Se caracteriza por la tríada clínica de estado mental alterado, hiperactividad autonómica y anormalidades neuromusculares ${ }^{17}$ y se clasifica según sus manifestaciones en leve (taquicardia, hipertensión, ansiedad, tremor, diarrea), moderado (fiebre y alteraciones neurológicas como hiperreflexia, clonus inducible o mioclonus con confusión o agitación psicomotora, rabdomiólisis asociada o no a acidosis metabólica o falla renal) o severo (hipertermia severa, hipertonía, convulsiones falla multisistémica entre ellas la respiratoria, somnolencia hasta coma) ${ }^{15}$. Las manifestaciones de este síndrome pueden atribuirse erróneamente a otra causa (accidentes cerebrovasculares, episodios convulsivos, delírium, intoxicaciones, síndrome neuroléptico maligno, etc.), puede llegar a ser desestimado o pasar desapercibido y no existe una prueba confirmatoria o hallazgos de laboratorio específicos para su diagnóstico; además, algunos medicamentos en asociación, como las benzodiazepinas pueden terminar enmascarando el cuadro $3,15,16,19$. 
Tabla 1. Medicamentos que precipitan el SS

\begin{tabular}{|c|c|}
\hline Mecanismo & Medicamentos relacionados \\
\hline Aumenta la formación de serotonina. & Triptófano \\
\hline Aumenta la liberación de serotonina & $\begin{array}{l}\text { Anfetaminas (incluyendo dextroanfetamina, metanfetamina) } \\
\text { Cocaína. } \\
\text { MDMA (éxtasis). } \\
\text { Derivados de la anfetamina (incluyendo fenfluramina, dexfenfluramina, fentermina). } \\
\text { Levodopa, carbidopa-levodopa (causa la liberación de serotonina de forma indirecta). }\end{array}$ \\
\hline $\begin{array}{l}\text { Afecta la recaptación de la hendidura sináptica a la } \\
\text { neurona presináptica }\end{array}$ & $\begin{array}{l}\text { Cocaína } \\
\text { MDMA (éxtasis) } \\
\text { Meperidina } \\
\text { Tramadol } \\
\text { Pentazocina } \\
\text { Inhibidores selectivos de la recaptación de serotonina (ISRS; incluidos citalopram, } \\
\text { escitalopram, fluoxetina, fluvoxamina, paroxetina y sertralina) } \\
\text { Inhibidores de la recaptación de serotonina y norepinefrina (IRSN, incluidos } \\
\text { desvenlafaxina, duloxetina, milnaciprán y venlafaxina) } \\
\text { Inhibidores de la recaptación de dopamina-norepinefrina (incluido el bupropión) } \\
\text { Moduladores de serotonina (incluyendo nefazodona, trazodona y vilazodona) } \\
\text { Antidepresivos tricíclicos ( incluyendo amitriptilina, amoxapina, clomipramina, } \\
\text { desipramina, doxepina, imipramina, maprotilina, nortriptilina, protriptilina, } \\
\text { trimipramina) } \\
\text { Hierba de San Juan (Hypericum perforatum) } \\
\text { Antagonistas del receptor 5-HT3 (incluyendo dolasetrón, granisetrón, ondansetrón, } \\
\text { palonosetrón) } \\
\text { Metoclopramida } \\
\text { Valproato } \\
\text { Carbamazepina } \\
\text { Sibutramina } \\
\text { Dextrometorfano } \\
\text { Ciclobenzaprina }\end{array}$ \\
\hline $\begin{array}{l}\text { Inhibe el metabolismo de la serotonina (es decir, inhibe } \\
\text { la actividad de la monoaminooxidasa) }\end{array}$ & $\begin{array}{l}\text { Inhibidores de la monoaminooxidasa (IMAO; incluyendo fenelzina, tranilcipromina, } \\
\text { isocarboxazida, moclobemida, safinamida, selegilina, rasagilina, linezolid, tedizolid, } \\
\text { azul de metileno, procarbazina) }\end{array}$ \\
\hline Agonista directo de la serotonina & $\begin{array}{l}\text { Buspirona } \\
\text { Triptanos (incluyendo sumatriptán, rizatriptán, otros) } \\
\text { Derivados ergóticos (incluyendo ergotamina, metilergonovina) } \\
\text { Fentanilo } \\
\text { Dietilamida del ácido lisérgico (LSD) }\end{array}$ \\
\hline Aumenta la sensibilidad del receptor postsináptico & Litio \\
\hline
\end{tabular}

Fuente: Modificada de Wang $R Z^{14}$ y Foong $A L^{16}$

El diagnóstico diferencial para SS incluye otros toxidromes como el síndrome neuroléptico maligno (SNM), la hipertermia maligna y la toxicidad anticolinérgica, así como afecciones como meningitis, encefalitis (en particular encefalitis autoinmune), hipertermia central e insolación'. El SNM es una reacción farmacológica idiosincrásica fulminante y potencialmente mortal a las dosis terapéuticas de los antagonistas de la dopamina ${ }^{3,4,20}$. Si bien el SS se caracteriza por hiperactividad neuromuscular (hiperreflexia con clonus, mioclono y temblor), en el SNM, generalmente hay una disminución de la actividad neuromuscular con características extrapiramidales (hipocinesia y rigidez en tubo de plomo), además que la hiperreflexia y el clonus en esta entidad rara vez se presentan ${ }^{21}$, contrario a la 
presencia de clonus en el SS; por otro lado el SNM se desarrolla durante días o semanas, mientras que el SS se desarrolla más rápidamente (generalmente dentro de las 24 horas) $)^{22}$. La hipertermia, la rigidez muscular, el estado mental alterado, la rabdomiólisis y los análisis de sangre alterados (leucocitosis, transaminasas hepáticas elevadas, creatina quinasa elevada y acidosis metabólica) pueden observarse en casos graves de ambos síndromes ${ }^{1}$. En la toxicidad anticolinérgica, el tono muscular y los reflejos son normales, con ruidos intestinales hipoactivos y piel seca y eritematosa; todas las características que ayudan a distinguirlo de SS'. La hipertermia maligna ocurre en individuos genéticamente susceptibles a los pocos minutos de la exposición a agentes anestésicos halogenados inhalados y relajantes musculares despolarizantes (como la succinilcolina); también se caracteriza por hipertermia, taquicardia, rigidez muscular tipo rigor-mortis, acidosis metabólica y concentraciones crecientes de dióxido de carbono ${ }^{3}$. Finalmente, los pacientes con infecciones del SNC o toxidromes simpaticomiméticos tienen menos probabilidades de exhibir este aumento de actividad ${ }^{23}$.

Los dos componentes principales para el manejo óptimo del SS son una buena atención de apoyo (especialmente en casos severos) y la evaluación de riesgos y el monitoreo cercano de aquellos con SS leve a moderado para evitar la progresión a SS severo que ponga en peligro la vida ${ }^{14}$. La primera trata de identificar y suspender medicamentos con acción serotoninérgica, cuidado de soporte para estabilizar los signos vitales (mantenimiento de las saturaciones de oxígeno, fluidos intravenosos y monitoreo cardíaco con registro continuo de la temperatura), sedación con benzodiazepinas (diazepam, midazolam o clonazepam), y en casos más graves, consideración de los antagonistas de la serotonina junto con la parálisis muscular1. Además del reconocimiento temprano, es esencial que el clínico reconozca la tasa de progresión potencialmente rápida del SS e inicie un tratamiento agresivo inmediato en aquellos que previamente fueron tratados de forma conservadora y que tienden a deteriorarse $e^{4,8}$.

Dentro del marco etiológico del SS, la asociación de medicamentos como el linezolid (inhibidor de la monoaminooxidasa) y el fentanilo (agonista directo de la serotonina) no ha sido ampliamente estudiada a la fecha, con pocos reportes de caso al respecto ${ }^{2,24,25,26}$. Estos dos medicamentos son en la actualidad utilizados con frecuencia tanto en el ámbito de infectología como en anestesiología (sedoanalgesia), por lo que es importante considerar que un paciente que reciba estos dos fármacos presenta riesgo potencial para desarrollar el SS, dado que la mortalidad por este síndrome que puede llegar hasta el 12\%27.

Se tiene conocimiento que los episodios de SS que involucran un inhibidor de la monoaminooxidasa pueden ser más graves y con mayor frecuencia conducir a resultados adversos, incluida la muerte ${ }^{14,28}$. En consecuencia el reconocimiento precoz, la alta sospecha clínica y tratamiento oportuno, sobre todo en un paciente crítico que esté bajo condiciones de sedoanalgesia, son esenciales para un adecuado desenlace, ya que en la mayoría de los casos, los síntomas generalmente se resuelven dentro de las 24 horas posteriores a la suspensión del medicamento, aunque en una minoría de los casos cuando el medicamento tiene metabolitos activos o una vida media más larga, los síntomas pueden durar mayor tiempo ${ }^{4}$.

El objetivo de este artículo es describir mediante la presentación de un caso clínico, cómo la coadministración y sinergismo entre fentanilo y linezolid actúa como entidad causante de SS (asociación poco informada en la literatura dentro de la etiología de este síndrome), desde las manifestaciones clínicas y el comportamiento paraclínico, ya que su diagnóstico representa un gran reto, sobretodo en la complejidad de un paciente crítico bajo condiciones de sedoanalgesia.

\section{Presentación de caso}

Motivo de consulta: "el paciente bajo efecto de sedoanalgesia (con midazolam y fentanilo) una vez que se inicia manejo antibiótico con linezolid, presenta una serie de manifestaciones sistémicas y neurológicas, condición que se exacerba y empeora posterior a la suspensión de la benzodiazepina".

Se trata de un paciente masculino de 21 años, con antecedente único de asma y alergia a la vancomicina, quien a su ingreso hospitalario (día 1) tuvo la necesidad de intubación orotraqueal por episodio de crisis asmática severa de difícil manejo con hipoxemia severa refractaria a manejo broncodilatador, por lo cual recibió sedación con midazolam $0.05 \mathrm{mg} / \mathrm{kg} / \mathrm{h}$ y fentanilo $1 \mathrm{mcg} / \mathrm{kg} / \mathrm{h}$ asociados con broncodilatadores (salbutamol y teofilina). Al cuarto día después de su ingreso, presentó picos febriles $\left(39.5^{\circ} \mathrm{C}, 38.5^{\circ} \mathrm{C}\right)$, secreciones 
orotraqueales purulentas y presencia de opacidades algodonosas en bases pulmonares con derrame pleural asociado documentado en la radiografía de tórax, compatible con neumonía broncoaspirativa complicada, por lo cual se inició manejo antibiótico con piperacilina-tazobactam 4.5 gr IV cada 6 horas con linezolid 600 mgIV cada 12horas. Los paraclínicos más sobresalientes se presentan en el día 4 de la tabla 2.

Posterior al inicio de la primera dosis de linezolid (6 horas después) se documentaron cifras tensionales elevadas (promedio 180/100), por lo que se inició manejo antihipertensivo con amlodipino a dosis de 10 mg día, sin control (promedio 180/95), agregándose posteriormente losartán a dosis de $50 \mathrm{mg}$ cada 12 horas con escasa mejoría de cifras (promedio 170/90), continuándose igual manejo a pesar de persistir con cifras elevadas pero sin criterio de urgencia ni emergencia hipertensiva. Cuatro días después se decidió extubar dada la evolución satisfactoria tanto clínica (mejoría de hipoxemia, de su patrón respiratorio, estabilidad hemodinámica y ausencia de signos de respuesta inflamatoria sistémica, al igual que de su broncoespasmo) como paraclínica (día 8 de la tabla 2), ordenándose la suspensión del goteo de sedoanalgesia instaurada (midazolam fentanilo).

A las 48 horas después de la suspensión de la sedación el paciente se tornó desorientado, hipertenso, taquicárdico y febril, sin interacción adecuada con el entorno, hiperquinético, con midriasis bilateral, falta de sostén cefálico, afasia global, diaforético y con episodios de diarrea. Al examen neurológico, además, hiperreflexia global, clónus, babinski derecho, con aumento del tono generalizado, sobre todo en extremidades izquierdas, acompañado de elevación de reactantes de fase aguda en paraclínicos que se le solicitó (día 10 de la tabla 2). Además, se tomó creatina-fosfocinasa (CPK) la cual se reportó elevada (2912 U/L) y una resonancia nuclear magnética (RNM) cerebral en la que no hubo alteraciones.

Tabla 2. Paraclínicos de ingreso y seguimiento del paciente

\begin{tabular}{|c|c|c|c|c|c|c|c|c|}
\hline Día de hospitalización & Día 4 & Día $8+$ & Día 10* & Día 11] & Día 12 & Día 13 & Día 14 & Unidades \\
\hline \multicolumn{9}{|l|}{ Hemograma } \\
\hline Leucocitos & 20.5 & 9.8 & 14.7 & 10.4 & & & & $10^{\wedge} 3 / \mathrm{ul}$ \\
\hline Neutrófilos & 16.9 & 5.6 & 10.4 & 3.4 & & & & $10^{\wedge} 3 / \mathrm{ul}$ \\
\hline Linfocitos & 2.5 & 1.2 & 1.6 & 1.4 & & & & $10^{\wedge} 3 / \mathrm{ul}$ \\
\hline Hemoglobina & 12.5 & 12 & 11 & 11.5 & & & & $\mathrm{~g} / \mathrm{dL}$ \\
\hline Hematocrito & 38.4 & 37.6 & 35 & 40 & & & & $\%$ \\
\hline Plaquetas & 537 & 190 & 455 & 220 & & & & $10^{\wedge} 3 / \mathrm{ul}$ \\
\hline Sodio & 142 & & 140 & 138 & & & & $\mathrm{mmol} / \mathrm{L}$ \\
\hline Fósforo & 4.4 & & 3.8 & 3.8 & & & & $\mathrm{mmol} / \mathrm{L}$ \\
\hline Calcio iónico & 1.147 & & 1.2 & 1.150 & & & & $\mathrm{mmol} / \mathrm{L}$ \\
\hline Magnesio & 2.1 & & 2.0 & 2.0 & & & & $\mathrm{mg} / \mathrm{dL}$ \\
\hline $\mathrm{Cl}$ & 102 & & 106 & 100 & & & & $\mathrm{mmol} / \mathrm{L}$ \\
\hline $\mathrm{Cr}$ & 0.73 & 0.71 & 1.36 & 1.0 & & & & $\mathrm{mg} / \mathrm{dl}$ \\
\hline Potasio & 4.2 & 4.71 & 4.9 & 4.3 & & & & $\mathrm{mmol} / \mathrm{L}$ \\
\hline Nitrógeno ureico & 10.2 & 8.2 & 22 & 15 & & & & $\mathrm{mg} / \mathrm{dl}$ \\
\hline PCR & 72 & 5.2 & 76 & 30.2 & & & & $\mathrm{mg} / \mathrm{L}$ \\
\hline CPK & & & 2912 & 1510 & 950 & 250 & 142 & $U / L$ \\
\hline
\end{tabular}

Día 8: paraclínicos de control posterior a 4 días de tratamiento antibiótico.

Día 10: día de inicio de sintomatología florida de SS y día suspensión de linezolid.

Día 11: primeras 24 horas después de la suspensión del linezolid.

Fuente: autores.

Con el cuadro anterior, habiendo descartado otras posibilidades diagnósticas, entre ellas las medicamentosas (síndrome neuroléptico maligno, hipertermia maligna o síndrome colinérgico) y las orgánicas (accidente cerebrovascular isquémico o hemorrágico u otras alteraciones estructurales), 
se realiza diagnóstico de SS asociado a la acción sinérgica del fentanilo y el linezolid, por lo que se suspendió el manejo antibiótico y se inició manejo con clonazepam.

Al día siguiente posterior a la suspensión del linezolid, se observó una disminución marcada de la CPK (día 11 de la tabla 2) así como de reactantes de fase aguda. A las 48 horas de la suspensión del Linezolid se evidenció mejoría clínica del paciente, con ausencia de signos neurológicos descriptivos del síndrome, favorable evolución con marcado descenso de la CPK, hasta el proceso de alta 96 horas después de suspensión del antimicrobiano, sin ninguna secuela hoy en día.

\section{Discusión}

El SS es una condición potencialmente mortal, asociada con una mayor actividad serotoninérgica tanto en el SNC como en el SNP, que requiere del reconocimiento precoz y la alta sospecha clínica por parte del personal de salud para evitar desenlaces fatales'; se reporta un paciente quien desarrolló este síndrome bajo condiciones de severidad, al ser expuesto inicialmente a fentanilo (como componente de sedoanalgesia) y 4 días después, a linezolid (por neumonía broncoaspirativa), quien inició con manifestaciones clínicas dentro de las primeras 24 horas después de haber iniciado el antimicrobiano. Estos son dos medicamentos que han sido considerados como entidades etiológicas del SS, el primero actuando como agonista directo de los receptores de serotonina y el segundo como inhibidor de la monoaminooxidasa ${ }^{6,7}$. Esta relación de fármacos actualmente es muy utilizada sobretodo en el contexto de pacientes críticos, pero muy poco referida en la literatura en cuanto a la etiología del SS.

No hay ensayos controlados aleatorizados o estudios de cohorte prospectivos que hayan examinado la tasa de toxicidad de la serotonina en pacientes que reciben linezolid y agentes serotoninérgicos. En los ensayos de fase III del linezolid de la Administración de Drogas y Alimentos de los Estados Unidos (FDA), entre 52 pacientes que tomaban linezolid y ISRS simultáneamente, no se informaron casos de SS $^{29}$. Lawrence et al. examinaron 2222 casos documentados de intoxicación serotoninérgica reportados al sistema de la FDA y encontraron 29 casos de toxicidad de serotonina asociada con linezolid; 13 de los cuales requirieron hospitalización y los fármacos concurrentes más frecuentes en estos casos fueron los ISRS, sin datos de la relación con el fentanilo ${ }^{24}$. Taylor y et al., en una revisión retrospectiva de casos en la Clínica Mayo (Rochester, Minnesota), encontraron una incidencia de SS del 3\% en pacientes que toman ISRS y linezolid ${ }^{30}$.

En búsqueda de la literatura se encuentran escasos reportes de casos clínicos de SS que asocian con linezolid y fentanilo, pero generalmente con asociación de otros medicamentos como los ISRS. Thomas et al, reportaron un caso de un paciente con quemaduras graves quien recibió los dos fármacos en cuestión, la fluoxetina desarrollando el síndrome una hora posterior al inicio de manejo antibiótico y con resolución a las 48 horas de suspensión de los fármacos serotoninérgicos ${ }^{31}$. Taylor et al, informaron dos casos clínicos, el primero un paciente con pseudoquiste pancreático quien inició las manifestaciones del síndrome dentro de las primeras 24 horas del inicio antibiótico, esta vez asociado con fentanilo y sertralina y con resolución de sintomatología 24 horas después de la suspensión del manejo antibiótico y el segundo con desenlaces similares en un paciente con infección del tracto urinario bajo manejo con venlafaxina, citalopram y fentanilo, asociado con linezolid ${ }^{32}$. Finalmente Strouse et al, quienes reportaron un caso clínico de un paciente con sarcoma metastásico quien a la asociación estudiada se adicionó duloxetina, desarrolló el síndrome 3 horas después del inicio de manejo antibiótico y presento resolución del cuadro 36 horas posterior al retiro de las medicaciones en cuestión ${ }^{33}$.

Con respecto al tiempo de inicio y duración del SS, Quinn et al en una revisión de literatura acerca de SS asociado con linezolid documentaron que el tiempo hasta el inicio de los síntomas varió entre menos de 24 horas hasta tres semanas de iniciado el manejo antibiótico, mientras que el tiempo hasta la resolución de los síntomas una vez que uno o ambos medicamentos fueron deprescritos varió de 1 a 5 días, siendo los de mayor asociación los ISRS 34 .

En el caso presentado observamos en primer lugar que la asociación entre linezolid y fentanilo mediante efecto sinérgico ( $\sin$ el uso de otros fármacos serotoninérgicos), pudo haber potenciado la aparición del SS 35 además en concordancia con la mayoría de los casos reportados anteriormente, se observa que las manifestaciones iniciaron 6 horas después del inicio del antimicrobiano (dentro de las primeras 24 horas), donde el paciente se tornó hipertenso con difícil control de cifras tensionales, 
como única manifestación. En este apartado se considera que el empleo de midazolam (como componente de la sedoanalgesia) pudo haber dificultado el diagnóstico inicialmente, al haber enmascarado la mayoría de las manifestaciones clínicas del síndrome ${ }^{2}$. 48 horas después de suspender sedoanalgesia en el proceso de extubación, el paciente comenzó a desarrollar la mayoría de las manifestaciones clínicas floridas del SS.

Son cuatro los principios fundamentales para el tratamiento del SS, entre ellos la deprescripción de todos los agentes serotoninérgicos, la atención de apoyo dirigida a la normalización de los signos vitales, la sedación con benzodiazepinas y la administración de antagonistas de la serotonina

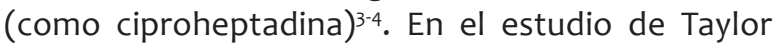
et al, los casos reportados de la asociación entre fentanilo, linezolid e ISRS, los cuadros mostraron mejoría sólo con la suspensión de la medicación implicada sin ninguna otra medida farmacológica adicional, sin embargo se denota que otros de los casos, en los cuales las manifestaciones fueron más severas (sobretodo asociación de linezolid y otros fármacos ISRS), hubo la necesidad de utilizar manejo adicional con benzodiazepinas y medicamentos como la ciproheptadina ${ }^{30}$. En el paciente, una vez confirmado el diagnóstico se decidió la suspensión del Linezolid (previamente se había suspendido el fentanilo) y se inició manejo con clonazepam, con notable mejoría clínica y paraclínica a partir de las 24 horas posteriores de la suspensión evidenciándose disminución progresiva y marcada de la CPK, de leucocitosis, neutrofilia, de pruebas de función renal y posteriormente mejoría clínica de los signos musculares, neurológicos, normalidad de signos vitales y síntomas neurovegetativos; lo que está acorde con la mayoría de los casos reportados de resolución de los síntomas una vez suspendida la medicación en cuestión ${ }^{31-34}$. De esta manera se sustenta y confirma el diagnóstico del paciente.

Dentro de los principales diagnósticos diferenciales que se descartaron en el paciente del caso, se consideraron la intoxicación por anticolinérgicos, que suele ser de inicio repentino, menor a horas, caracterizada por reflejos y tono muscular normales, mucosas secas, piel eritematosa, caliente y seca, retención urinaria, ausencia de ruidos gastrointestinales, midriasis, excitación, delirio, hipertensión, taquicardia, taquipnea e hipertermia'. Los ruidos hidroaéreos aumentados, hiperreflexia, sudoración, y el color de piel normal, distinguen al SS de la intoxicación por anticolinérgicos².

Por otro lado, la hipertermia maligna es una alteración farmacogenética caracterizada por hipertonicidad marcada, hiporreflexia y acidosis metabólica, habitualmente ocurre de minutos a horas luego de la exposición a agentes anestésicos inhalatorios, la presencia de rigidez extrema e hiporreflexia la distinguen del $\mathrm{SS}^{3,4}$, no siendo el caso del paciente.

Por último, el síndrome neuroléptico maligno que se caracteriza por su inicio lento, bradiquinesia o aquinesia, rigidez, fluctuaciones en el nivel de conciencia e inestabilidad autonómica, piel pálida y sudorosa, sialorrea, pupilas y ruidos gastrointestinales normales, en contraste con el SS donde se presenta hiperquinesia de comienzo súbito que lo diferencia del síndrome neuroléptico 2,4,18,36.

Otros diagnósticos para tener en cuenta son la sepsis, la encefalopatía herpética, el delirium tremens, el golpe de calor, la tormenta tiroidea, la intoxicación por salicilatos, el infarto agudo de miocardio, entidades que se presentan con hipertermia, o en forma de paroxismos o crisis, y la intoxicación con agentes adrenérgicos ${ }^{2,4,37}$, clínica no compatible con la del caso clínico. En el paciente se descartó cualquier alteración estructural como accidente cerebrovascular sea isquémico o hemorrágico, por medio de las neuroimágenes.

\section{Conclusión}

El SS es una entidad cuyo diagnóstico depende del conocimiento y la alta sospecha clínica por parte del personal de salud, en un paciente que reciba medicamentos que actúen sobre el metabolismo de la serotonina. Concluimos que la administración concomitante de fentanilo y linezolid puede favorecer la aparición del SS, mientras que el uso de benzodiazepinas puede enmascarar el cuadro dificultando su diagnóstico.

\section{Conflicto de intereses}

Los autores declaran no tener ningún conflicto de intereses. 


\section{Referencias bibliográficas}

1. Scotton WJ, Hill LJ, Williams AC, Barnes NM. Serotonin Syndrome: Pathophysiology, Clinical Features, Management, and Potential Future Directions. Int J Tryptophan Res [Internet]. 2019 Sep 9;12:1178646919873925. Disponible en: $10.1177 / 1178646919873925$.

2. Mason PJ, Morris VA, Balcezak TJ. Serotonin syndrome. Presentation of 2 cases and review of the literature. Medicine (Baltimore) [Internet]. 2000 Jul;79(4):201-9. Disponible en: 10.1097/00005792-200007000-00001.

3. Jurek L, Nourredine M, Megarbane B, d'Amato T, Dorey JM, Rolland B. The serotonin syndrome: An updated literature review. Rev Med Interne [Internet]. 2019 Feb;40(2):98-104. Disponible en: 10.1016/j.revmed.2018.08.010.

4. Boyer EW, Shannon M. The serotonin syndrome. N Engl J Med [Internet]. 2005 Mar 17;352(11): 1112-20. Disponible en: 10.1056/ NEJMra041867.

5. Bartlett D. Drug-Induced Serotonin Syndrome. Crit Care Nurse [Internet]. 2017 Feb;37(1):49-54. Disponible en: 10.4037/ ccn2017169.

6. Karkow DC, Kauer JF, Ernst EJ. Incidence of Serotonin Syndrome With Combined Use of Linezolid and Serotonin Reuptake Inhibitors Compared With Linezolid Monotherapy. J Clin Psychopharmacol [Internet]. 2017 Oct;37(5):518-23. Disponible en: 10.1097/JCP.0000000000000751.

7. Foong AL, Grindrod KA, Patel T, Kellar J. Demystifying serotonin syndrome (or serotonin toxicity). Can Fam Physician. 2018;64(10):720-27

8. Sternbach H. The serotonin syndrome. Am J Psychiatry 1991;148(6):705-13

9. Mojtabai R, Olfson M. National trends in long-term use of antidepressant medications. J Clin Psychiatry. 2014;75(2):169-177.

10. Nguyen CT, Xie L, Alley S, McCarron RM, Baser O, Wang Z. Epidemiology and economic burden of serotonin syndrome with concomitant use of serotonergic agents: A retrospective study utilizing two large US claims databases. Prim Care Companion CNS Disorder. 2017;19(6).

11. Watson WA, Litovitz TL, Klein-Schwartz W, Rodgers GC, Reid N, Youniss J, et al. 2004 Annual Report of the American Association of Poison Control Centers Toxic Exposure Surveillance System. Am J Emerg Med. 2005;23(5):589-666.

12. Watson WA, Litovitz TL, Rodgers GC Jr, Klein-Schawartz, Youniss J, Rose SR. 2002 Annual Report of the American Association of Poison Control Centers Toxic Exposure Surveillance System. Am J Emerg Med. 2003;21(5):353-421.

13. Gummin DD, Mowry JB, Spyker DA, Brooks DE, Fraser MO, Banner W. Annual report of the American Association of Poison Control Centers' National Poison Data System (NPDS): 34th annual report. Clin Toxicol. 2017;55(10):1072-1252.

14. Isbister G, Buckley N. The pathophysiology of serotonin toxicity in animals and humans: implications for diagnosis and treatment. Clin Neuropharmacol. 2005;28(5):205-14

15. Werneke U, Jamshidi F, Taylor D, Ott M. Conundrums in neurology: diagnosing serotonin syndrome - a meta-analysis of cases. BMC Neurol. 2016;16:97

16. Mackay FJ, Dunn NR, Mann RD. Antidepressants and the serotonin syndrome in general practice. $\mathrm{Br} J$ Gen Pract. 1999;49(448):871-874.
17. Francescangeli J, Karamchandani K, Powell M, Bonavia A. The Serotonin Syndrome: From Molecular Mechanisms to Clinical Practice. Int J Mol Sci. 2019;20(9):2288.

18. Ener RA, Meglathery SB, Van Decker WA, Gallagher RM. Serotonin syndrome and other serotoninergic disorders. Am Acad of Pain Med. 2003; 4(1): 63-74.

19. 19. Volpi-Abadie J, Kaye AM, Kaye AD. Serotonin syndrome. Ochsner J. 2013;13(4):533-540.

20. Dosi R, Ambaliya A, Joshi H, Patell R. Serotonin syndrome versus neuroleptic malignant syndrome: a challenging clinical quandary. BMJ Case Rep. 2014;2014(1):bcr2014204154.

21. Mills KC. Serotonin syndrome. A clinical update. Crit Care Clin. 1997;13(4):763-783.

22. Dunkley EJC, Isbister GK, Sibbritt D, Dawson AH, Whyte IM. The Hunter Serotonin Toxicity Criteria: simple and accurate diagnostic decision rules for serotonin toxicity. QJM. 2003;96(9):635-642.

23. Boyer EW, Traub SJ, Grayzel J. Serotonin Syndrome. In: UpToDate, Waltham, MA, 2010.

24. Lawrence K, Adra M, Gillman P. Serotonin Toxicity Associated with the Use of Linezolid: A Review of Postmarketing Data. Clin Infect Dis. 2006;42(11), 1578-1583.

25. Mandell L, Bennett E, Dolin R: Principles and Practice of Infectious Diseases, 5th edition. Churchill Livingstone, Inc. 2000. pp. 392-393

26. Samartzis L, Savvari P, Kontogiannis S, Dimopoulos S. Linezolid Is Associated with Serotonin Syndrome in a Patient Receiving Amitriptyline, and Fentanyl: A Case Report and Review of the Literature. Case Rep Psychiatry. 2013;2013(3): 617251.

27. Frank, Christopher. Recognition and treatment of serotonin syndrome. Frank C. Recognition and treatment of serotonin syndrome. Can Fam Physician. 2008;54(7):988-992.

28. Bronstein A, Spyker D, Cantilena L, Rumack B, Dart R. Annual report of the American Association of Poison Control Centers' National Poison Data System (NPDS): 29th Annual Report. Clin Toxicol (Phila). 2012; 50(10): 911 - 1164.

29. Rubinstein E, Isturiz R, Standiford HC, Smith LG, Oliphant TH, Cammarata S et al. Worldwide assessment of linezolid's clinica safety and tolerability: comparator-controlled phase III studies. Antimicrob Agents Chemother. 2003;47(6):1824-1831.

30. Taylor JJ, Wilson JW, Estes LL. Linezolid and serotonergic drug interactions: a retrospective survey. Clin Infect Dis. 2006;43(2):180-187.

31. Thomas CR, Rosenberg M, Blythe V, et al. Serotonin syndrome and linezolid. J Am Acad Child Adolesc Psychiatry. 2004;43(7):790.

32. Taylor JJ, Estes LL, Wilson JW. Linezolid and serotonergic drug interactions. Clin Infect Dis. 2006;43(2):180-187.

33. Strouse TB, Kerrihard TN, Forscher CA, et al. Serotonin syndrome precipitated by linezolid in a medically ill patient on duloxetine. J Clin Psychopharmacol. 2006;26(6):681-683.

34. Quinn DK, Stern TA. Linezolid and serotonin syndrome. Prim Care Companion J Clin Psychiatry. 2009;11(6):353-356.

35. Ramsay R, Dunford C, Gillman P. Methylene blue and serotonin toxicity: inhibition of monoamine oxidase A (MAO A) confirms a theoretical prediction. Br J Pharmacol. 2007;152(6): 946 - 951.

36. Birmes P, Coppin D, Schmitt L, Lauque D. Serotonin syndrome: brief review. CMAJ. 2003;168(11):1439-1442.

37. Young P, Finn BC, Pellegrini D, Soloaga ED, Bruetman JE. Tormenta simpatica paroxistica siguiendo a injuria axonal difusa. Medicina (B. Aires). 2006;66(5): 439-42. 